\title{
Uncovering the evolutionary origin of plant molecular processes: comparison of Coleochaete (Coleochaetales) and Spirogyra (Zygnematales) transcriptomes
}

\author{
Ruth E Timme*1 and Charles F Delwiche1,2
}

\begin{abstract}
Background: The large and diverse land plant lineage is nested within a clade of fresh water green algae, the charophytes. Collection of genome-scale data for land plants and other organisms over the past decade has invigorated the field of evolutionary biology. One of the core questions in the field asks: how did a colonization event by a green algae over 450 mya lead to one of the most successful lineages on the tree of life? This question can best be answered using the comparative method, the first step of which is to gather genome-scale data across closely related lineages to land plants. Before sequencing an entire genome it is useful to first gather transcriptome data: it is less expensive, it targets the protein coding regions of the genome, and provides support for gene models for future genome sequencing. We built Expressed Sequence Tag (EST) libraries for two charophyte species, Coleochaete orbicularis (Coleochaetales) and Spirogyra pratensis (Zygnematales). We used both Sanger sequencing and next generation 454 sequencing to cover as much of the transcriptome as possible.

Results: Our sequencing effort for Spirogyra pratensis yielded 9,984 5' Sanger reads plus 598,460 GS FLX Standard 454 sequences; Coleochaete orbicularis yielded 4,992 5' Sanger reads plus 673,811 GS FLX Titanium 454 sequences. After clustering S. pratensis yielded 12,000 unique transcripts, or unigenes, and C. orbicularis yielded 19,000. Both transcriptomes were very plant-like, i.e. most of the transcripts were more similar to streptophytes (land plants + charophyte green algae) than to other green algae in the sister group chlorophytes. BLAST results of several land plant genes hypothesized to be important in early land plant evolution resulted in high quality hits in both transcriptomes revealing putative orthologs ripe for follow-up studies.

Conclusions: Two main conclusions were drawn from this study. One illustrates the utility of next generation sequencing for transcriptome studies: larger scale data collection at a lower cost enabled us to cover a considerable portion of the transcriptome for both species. And, two, that the charophyte green algal transcriptoms are remarkably plant-like, which gives them the unique capacity to be major players for future evolutionary genomic studies addressing origin of land plant questions.
\end{abstract}

\section{Background}

The ancestry of all living land plants (embryophytes) can be traced back to a single colonization event from a charophyte green alga. In other words, the tremendous diversity we see in land plants today--from mosses to redwoods and orchids--all descended from a single common

* Correspondence: retimme@umd.edu

${ }^{1}$ Cell Biology and Molecular Genetics, University of Maryland, College Park, MD, 20742, USA

Full list of author information is available at the end of the article ancestor that colonized land 430-470 million years ago $[1,2]$. Uncertainty remains concerning the precise relationships between embryophytes and their algal relatives [3-8], but there is no serious doubt that the origin of land plants occurred from within the charophytes. There are six orders of charophyte green algae that, when embryophytes are included, comprise the Streptophyta sensu lato (s.l.) [9]: the Mesostigmatales, Chlorokybales, Klebsormidiales, Zygnematales, Coleochaetales and Charales. 
Both phylogenetic and fossil evidence suggest that these orders are extremely old lineages, comparable in age to the land plants [2]. Therefore, an understanding of the biology of land plants based on comparative genomics would benefit greatly if data were available from these organisms. Unfortunately, in most cases the genome size is poorly characterized, the tools of molecular genetics are not well developed, or cultures are difficult to maintain. Consequently, the acquisition of genomic data from these organisms has lagged other lineages. To move toward comprehensive genomic analysis of charophytes, we undertook EST analysis of two representative charophytes, Spirogyra pratensis and Coleochaete orbicularis.

Despite there being significant genomic resources available for the broader group of green algae, including Chlorophyta, there is only one published EST library to date that directly bears on the charophytes, that of Mesostigma viride [10]. Mesostigma is a unicellular, monotypic genus that in some analyses is placed as sister to the rest of streptophytes [6,11-14], although other studies have placed it as a sister to all other green algae $[15,16]$. In either case, its EST library is a valuable resource for this study. Most taxonomic and ecological diversity in the green algae resides in the Chlorophyta, a large clade sister to the streptophytes. Among the important organisms in this sister clade are the model organism Chlamydomonas reinhardtii and the ecologically significant Ostreococcus tauri. Both of these organisms have fully sequenced and published genomes $[17,18]$.

According to Darwin's centralizing theme of descent with modification, one would predict that all land plant genes should have a homolog in the charophytes unless there was horizontal gene transfer from a non-plant organism, or unprecedented neofunctionalization. However, any one lineage of charophytes might be expected to have lost or modified some of these in the 500 million years or more of independent evolutionary history that separates each lineage from embryophytes. In this context, it is to be expected that land plant genes and their associated molecular pathways either originated in the charophytes or, if more ancient, were retained along these green algal lineages leading up to the colonization of land. Thus, it is important to sample broadly among the charophytes if the homologs of key embryophyte genes are to be identified.

In recent years PCR-based approaches have been used to fish out specific land plant genes of interest in the charophyte lineages, but advances in sequencing technologies have now made it far more efficient to gather highthroughput genomic data and work backwards, using plant gene models to annotate the putative homologous genes. Sequencing expressed sequence tags (ESTs) is an efficient first pass at gathering a large portion of the genomic coding regions. We undertook here an analysis of two distantly related charophyte taxa: Spirogyra pratensis Transeau (Zygnematales) and Coleochaete orbicularis Pringh. (Coleochaetales). Both of these lineages are essential to understanding the placement of land plants in the context of their nearest living green algal relatives. In addition, evidence of land plant molecular pathways, such as the ethylene response pathway, in the charophytes would reveal the origins of key plant molecular processes.

\section{Results}

\section{EST statistics}

Our sequencing effort for Spirogyra pratensis yielded 9,984 5' Sanger reads plus 598,460 GS FLX Standard 454 sequences; Coleochaete orbicularis yielded 4,992 5' Sanger reads plus 673,811 GS FLX Titanium 454 sequences (Table 1). The average length of Sanger sequences was 915 bp (C. orbicularis) or 1,346 bp (S. pratensis) before trimming for low quality and vector sequence. The average length for the raw 454 reads differed between the older GS FLX Standard and newer GS FLX Titanium sequencing technologies of 211 and 378 bp, respectively. The 454 sequences for each species were trimmed of vector and low-quality sequences, and then

Table 1: EST Sequence statistics

\begin{tabular}{|c|c|c|c|c|c|}
\hline & 454 reads & 5' Sanger reads & 454 assembly & Sanger assembly & Combined assembly \\
\hline \multicolumn{6}{|l|}{ C. orbicularis } \\
\hline Number of reads & 673,811 & 4992 & 26,373 & 2,455 & 19,313 \\
\hline Average length (bp) & 378 & 915 & 712 & 721 & 813 \\
\hline GC content & $47.9 \%$ & $46.4 \%$ & $49.2 \%$ & $48.6 \%$ & $49.4 \%$ \\
\hline \multicolumn{6}{|l|}{ S. pratensis } \\
\hline Number of reads & 598,460 & 9984 & 12,357 & 2836 & 12,191 \\
\hline Average length (bp) & 211 & 1346 & 493 & 845 & 571 \\
\hline GC content & $42.7 \%$ & $43.9 \%$ & $41.1 \%$ & $42.5 \%$ & $41.1 \%$ \\
\hline
\end{tabular}


clustered by Agencourt. The 454 assembly along with the raw Sanger reads were then clustered together using our in-house pipeline (Figure 1). This two-stage clustering method reduced the demand on computational power for the final assembly (CAP3 run on a desktop with $10 \mathrm{~GB}$ of RAM was not enough memory to cluster all the raw data together). It also allowed for careful tracking of each sequencing effort contributing to the final assembly. The average length of unigenes (contigs + singletons) in the combined 454 and Sanger sequence assembly was $813 \mathrm{bp}$ for C. orbicularis and 571 for S. pratensis.

Summarizing the composition of the clustered ESTs is complex due to our two-step assembly procedure (the 454-only assembly followed by a combined $454+$ Sanger assembly) (Table 2). Results from the Coleochaete orbicularis CAP3 assembly produced 19313 unigenes: 3,854 contigs and 1,549 singletons with the average length of contigs at 1,313 bp and singletons at less than half of that at $688 \mathrm{bp}$. The Spirogyra pratensis-combined assembly resulted in 12,191 unigenes: 1,707 contigs (average length $1,265 \mathrm{bp}$ ) and 10,490 singletons (average length $458 \mathrm{bp}$ ). The singletons category actually comprised several types of data: 454-only contigs from the first assembly, single 454 reads and single Sanger reads. Most of the singletons were in fact 454-only contigs for both EST libraries (numbers in Table 2). The number of reads per unigene was low on average, with the vast majority of unigenes comprising only one to five reads (Figure 2). A few contigs had higher numbers of reads, the most numerous being one $\mathrm{S}$. pratensis contig with 258 reads.

Because ESTs are, by definition, sequences derived from messenger RNA, it follows that they should all contain at least a portion of protein-coding sequence, but several forms of contamination are possible. Our sequencing effort included 5' Sanger sequencing plus 454 sequencing, which is generated from sequencing shorter reads from sheared cloned inserts. Due to this strategy, we expected to recover the $5^{\prime}$ (and possibly the $3^{\prime}$ ) UTR and some portion of the coding sequence. However, in addition to mRNA, there is a chance of contamination in the EST library from non-coding nucleic acids, such as

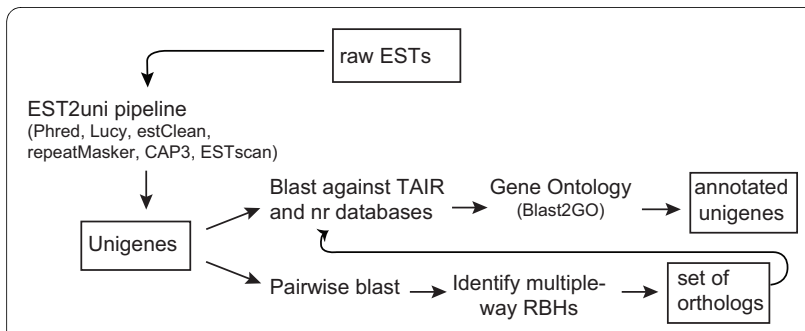

Figure 1 EST analysis pipeline. Diagram showing steps in the EST analysis pipeline. Perl-scripts were written by author unless otherwise noted. 'Raw ESTs' refer to the Sanger chromatograms plus the first clustering pass of the 454 sequences.
rRNA, tRNA and genomic DNA. These contaminants were removed from the final unigene set after ESTscan determined the putative coding regions in the sequences. By definition only the putative protein coding sequences were included in the final unigene set. Out of 12,191 total unigenes in Spirogyra pratensis, 25\%, or 2,076, had no coding region detected. Coleochaete orbicularis had a lower percentage of non-coding contaminants, at $16 \%$, or 3,080 sequences in which no protein-coding region was detected.

\section{Taxonomic Assignment}

Although every attempt was made to produce axenic cultures, including streaking and isolation off agar plates, antibiotic treatments, and zoospore isolation, we settled on "very clean" cultures. Because of this we anticipated a few contaminants based on low bacterial contamination observed when we grew the culture on agar plates. For this reason, the unigenes (all nucleotide sequences for the unique contigs and singletons) were BLASTed against the NCBI non-redundant (nr) nucleotide database using BLASTx for taxonomic lineage assignment. Eight major taxonomic categories were recovered as top hits for each Spirogyra pratensis and Coleochaete orbicularis: Archaea, Viruses, Bacteria, Fungi, Chlorophyta, Streptophyta, various other Eukaryotes, and unigenes with no significant hits (Figure 3). The proportion of hits for each species was very similar and therefore will be summarized together. About $10 \%$ of hits were possible contaminants (Bacteria, Fungi and various other Eukaryotes) and roughly $2-3 \%$ were hits to the other lineage of green algae, Chlorophyta (several chlorophyte genomes were present in GenBank at the time of this analysis). The remaining $\sim 85 \%$ were split fairly evenly between the top hit being a streptophyte (mostly land plants) or no hits returned at all (e-value $<0.0001$ ). A significant percentage of ESTs received no hits at all: $41 \%$ and $48 \%$ for S. pratensis and C. orbicularis, respectively. In this class of unknown unigenes, a significant portion of the sequences did not contain a protein prediction using ESTScan [19]. About half of the Spirogyra "no hits" and one-third in the Coleochaete "no hits" category were not included in the predicted protein set of unigenes, which means they are most likely a mixture of genomic and non-coding RNA contaminants. That leaves about $20 \%$ and $28 \%$ of putative novel genes in S. pratensis and C. orbicularis, respectively.

Because GenBank does not have uniform taxon sampling across the tree of life, it is important not to place too much significance on the BLAST based lineage assignment for the unigenes. For example, a small fraction of hits to metazoan taxa (included in the "various other Eukaryotes" category) most likely represents either a conserved protein domain or an ancestral gene lost in land plants, and not a metazoan contaminant. Likewise, 
Table 2: Contig assembly statistics. Combined 454+Sanger contig assembly statistics.

\begin{tabular}{|c|c|c|c|c|c|}
\hline & \multirow[t]{2}{*}{ CAP3 Contigs } & \multicolumn{4}{|c|}{ Unassembled 'singletons' } \\
\hline & & Total & 454-only contigs & 454 single reads & Sanger only \\
\hline \multicolumn{6}{|l|}{ C. orbicularis } \\
\hline Number of reads & 3854 & 15459 & 14919 & 40 & 500 \\
\hline Average length (bp) & 1313 & 688 & & & \\
\hline \multicolumn{6}{|l|}{ S. pratensis } \\
\hline Number of reads & 1707 & 10490 & 9425 & 0 & 1065 \\
\hline Average length (bp) & 1265 & 458 & & & \\
\hline
\end{tabular}

BLAST hits to bacteria and fungi, unless evaluated individually, should be treated with caution. On the contrary, this means that up to $10 \%$ of the ESTs from each of these species could be derived from bacterial and fungal contaminants and not from the charophyte for which it's assigned. The analyses in this manuscript were largely focused on the unigenes that had similarity to land plant genomes, so it is unlikely that contaminant unigenes affected any of our major conclusions.

\section{Orthologous Genes}

The results of the lineage assignment introduced the question of how many genes in each EST library are actually shared with chlorophytes vs. streptophytes. To address this question, the protein predicted unigenes for Coleochaete orbicularis and Spirogyra pratensis, plus complete proteins from Chlamydomonas reinhardtii (a chlorophyte) and Arabidopsis thaliana (a streptophyte) were included in an all-vs-all pair-wise BLASTp. The reciprocal best hits (RBHs) between any two, three or four species were interpreted to be orthologs if all pairwise possibilities in the ortholog set were each other's reciprocal best hit. All $\mathrm{RBH}$ overlaps are displayed in a Venn diagram (Figure 4). The total number of unigenes (or, proteins, in the case of the two reference genomes) for each species can be obtained by adding up all the numbers in the respective ovals. For example, a set of 718 unigenes are RBHs for all four species. A different set of 114 unigenes are RBHs for the charophytes + Chlamydomonas, but not for Arabidopsis.

Although there are many overlaps to report, the most interesting results from this analysis concerns the overlap between our species of interest and its overlap with chlorophytes $v s$. streptophytes. Looking at pair-wise comparisons only, C. orbicularis shares 1,707 orthologs with Arabidopsis vs. 562 with Chlamydomonas. Spirogyra pratensis has a similar pattern, with 1,030 shared Arabidopsis orthologs and 318 shared Chlamydomonas orthologs. This pattern holds up with three-way RBHs: $C$. orbicularis + S. pratensis share 553 orthologs with Arabi- dopsis verses 114 with Chlamydomonas. (A list of RBHs shared by the charophytes + Chlamydomonas are included in Additional File 1.)

A list of genes hypothesized to be important in early land plant colonization, or "green genes," was generated from Graham et al. [20]. Each of these genes had at least one hit between the two libraries, except for the MADS domain, which had no hits in either library (Table 3). The following genes had at least one hit in both Coleochaete orbicularis and Spirogyra pratensis: RSW1 (cellulosic cell wall), GEM1/MOR1 (cytokinetic phragmoplast), CTR1 (plasmodesmata), and MERISTEM LAYER1 (multicellular sporophyte body). WUSCHEL and GNOM (asymmetric cell division) were only found in C. orbicularis and the expansins were only found in S. pratensis. A moss (Physcomitrella patens) and a chlorophyte (Chlamydomonas reinhartii) were included as references. Although all of these are significant BLAST "hits" (e-value < 1e-20) they can only be interpreted as genes of interest. Any orthology determination would need a more vigerous follow-up analysis.

\section{Gene Ontology}

The Gene Ontology (GO) categories recovered from Blast2GO analyses were summarized by the proportion of unigenes annotated in each GO level 3 category (Figure 5). The complete set of proteins for Arabidopsis thaliana was included in the analysis as a relative proportional measure since the GO assignments were all derived from A. thaliana annotations. Overall, Spirogyra pratensis and Coleochaete orbicularis unigene sets were remarkably similar in relative proportions, but they deviated from $A$. thaliana in several categories. Three categories stood out in biological processes (BP): both green algae were underrepresented in cellular metabolic process and regulation of biological quality ( $\mathrm{p}<0.001 ; \mathrm{p}<0.001$ for $S$. pratensis and $C$. orbicularis respectively), but they were overrepresented in the biosynthetic processes category $(p<0.001$; $\mathrm{p}<0.001)$. For molecular function $(\mathrm{MF})$, the green algae unigenes were overrepresented in transferase activity $(\mathrm{p}<$ 
a.)

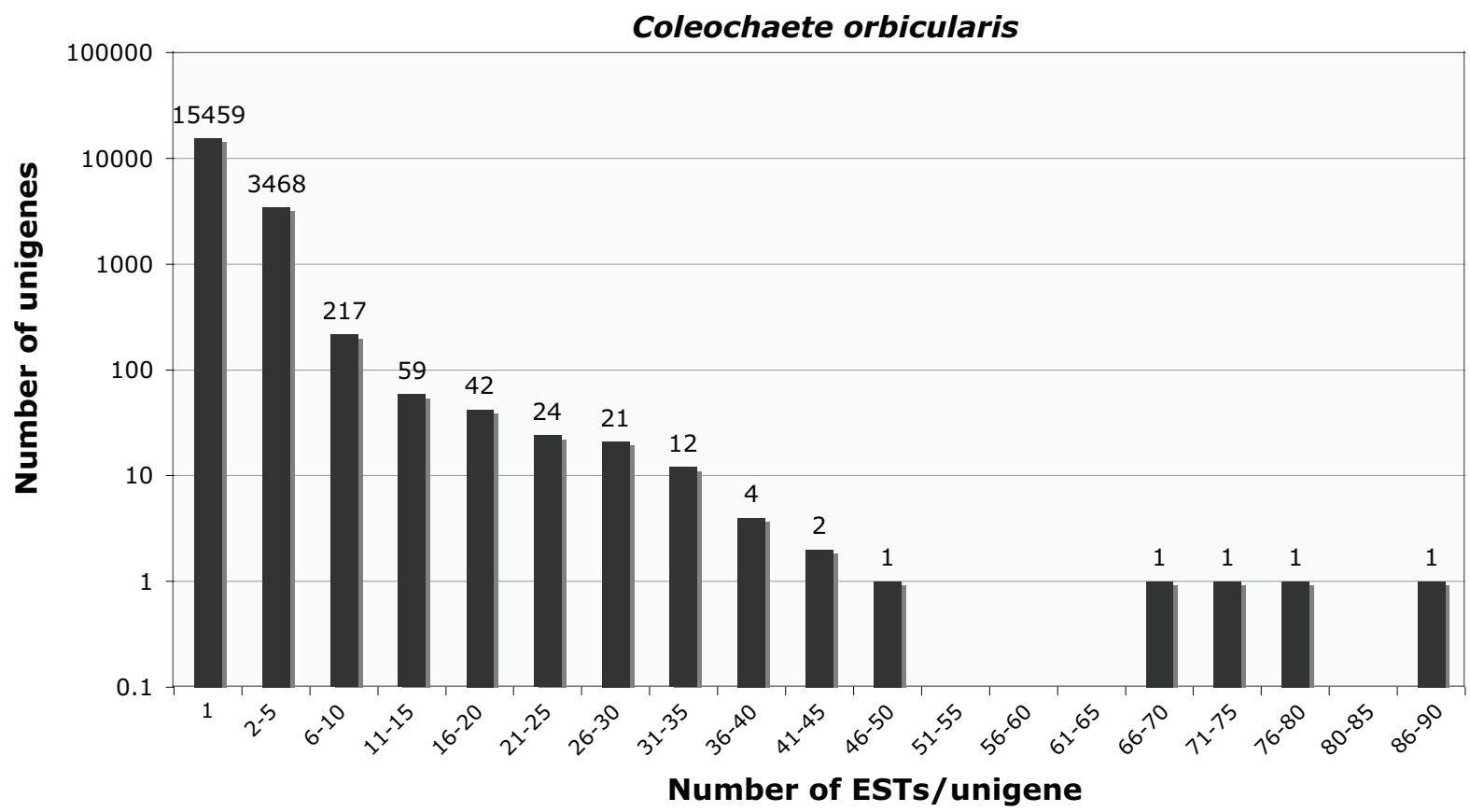

b.)

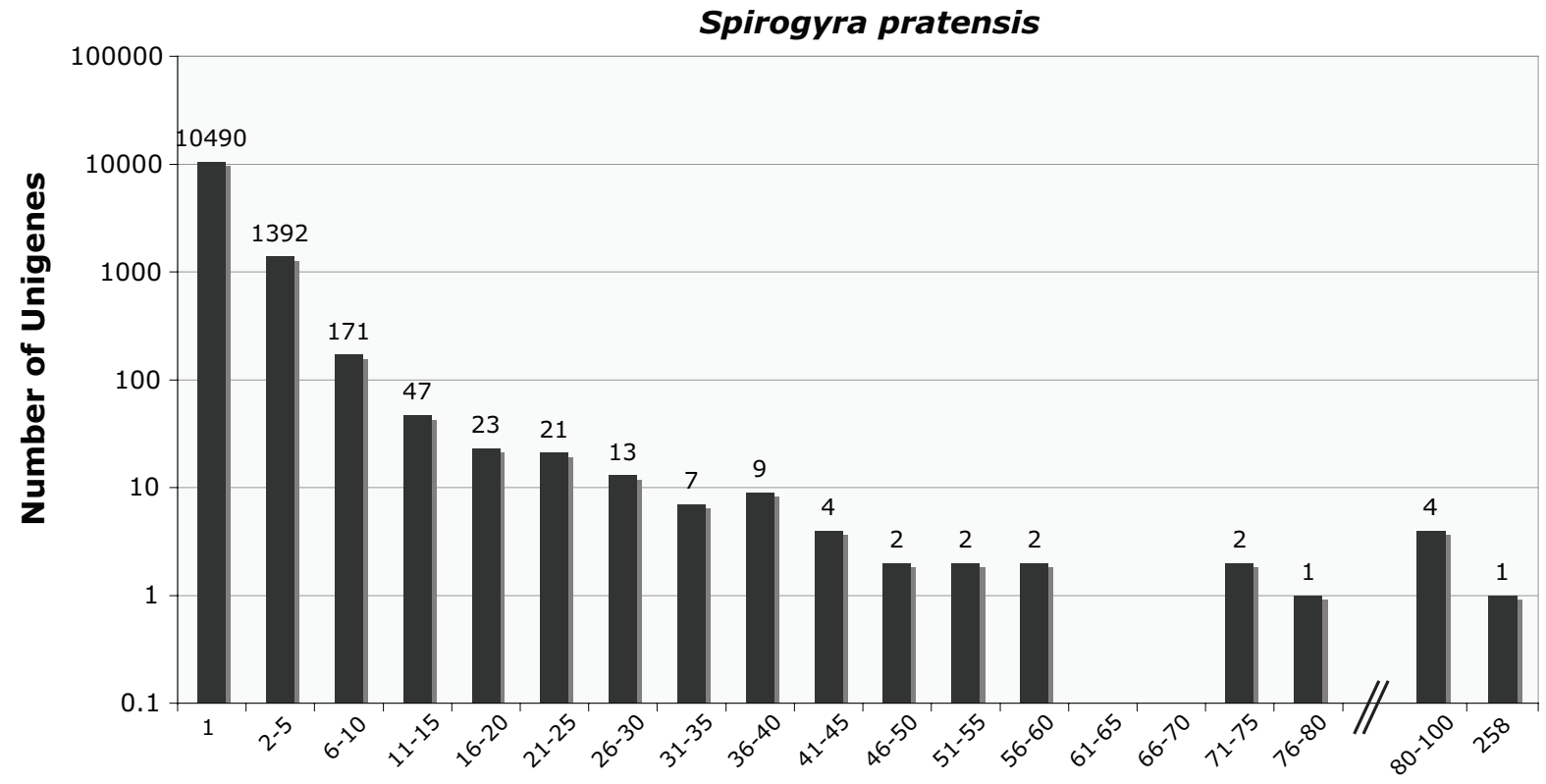

Number of ESTs/unigene

Figure 2 EST Cluster Analysis. Cluster analysis for a.) Coleochaete orbicularis and b.) Spirogyra pratensis in log scale. Each graph shows the number of ESTs per unigene. Most of the unigenes in each EST library contain a single or low number of redundant ESTs.

0.001; $\mathrm{p}<0.001)$, hydrolase activity $(\mathrm{p}<0.001 ; \mathrm{p}<0.001)$ and nucleotide binding ( $\mathrm{p}<0.001 ; \mathrm{p}<0.001)$, but they were underrepresented in the transcription factor activity $(\mathrm{p}<0.001 ; \mathrm{p}<0.001)$ and in oxidoreductase activity categories $(\mathrm{p}<0.00 ; \mathrm{p}<0.001)$. For cellular compartment
(CC) annotations, the green algae unigenes were slightly over-represented in many categories, but deviated most drastically in their underrepresentation in membrane and intracellular organelle part categories $(\mathrm{p}<0.001$; $\mathrm{p}<<$ 0.001). 


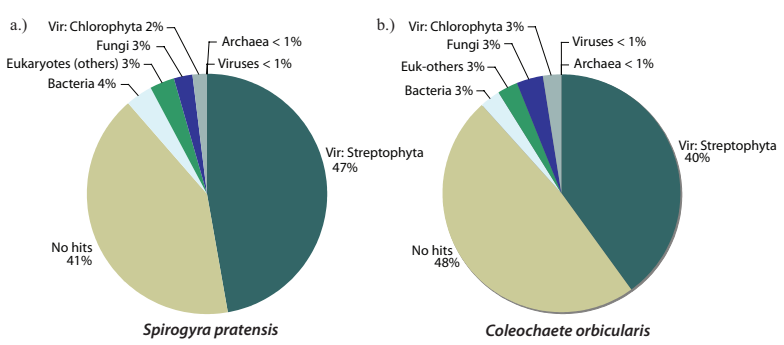

Figure $\mathbf{3}$ Lineage information. Summary of the lineage information extracted from the top BLASTx hit to NCBI's non-redundant protein database. Vir = viridiplantae. In-house perl scripts were used to query Entrez for taxonomy information.

\section{Discussion}

Both charophyte EST libraries are very land plant-like. More specifically, most of the transcriptome is more similar to embryophytes (land plants) than to other green algae in the chlorophyte lineage (the other green algal lineage sister to the streptophytes). There are some exceptions, however, which are interesting. Looking just at BLAST hits against NCBI's non-redundant (nr) protein database, 2\% (214 unigenes) and 3\% (488 unigenes) of the transcriptome had a top hit to a chlorophyte in Spirogyra and Coleochaete, respectively. While this is only a small fraction of total unigenes, it suggests possible shared ancestral genes between the chlorophyte and streptophyte lineages that were lost along the land plant lineage. For example, there are flagellar-associated proteins in both Spirogyra (two) and Coleochaete (13) that have top BLAST hits to Chlamydomonas and Ostreococcus, but that have no hits to Arabidopsis or any other land plant. Plants lost their flagellate cells in the Gymnosperms, which left Angiosperms with no motile cells. It follows that the flagellar-associated proteins would also be lost in flowering plants, infact, analysis of the much more ances-

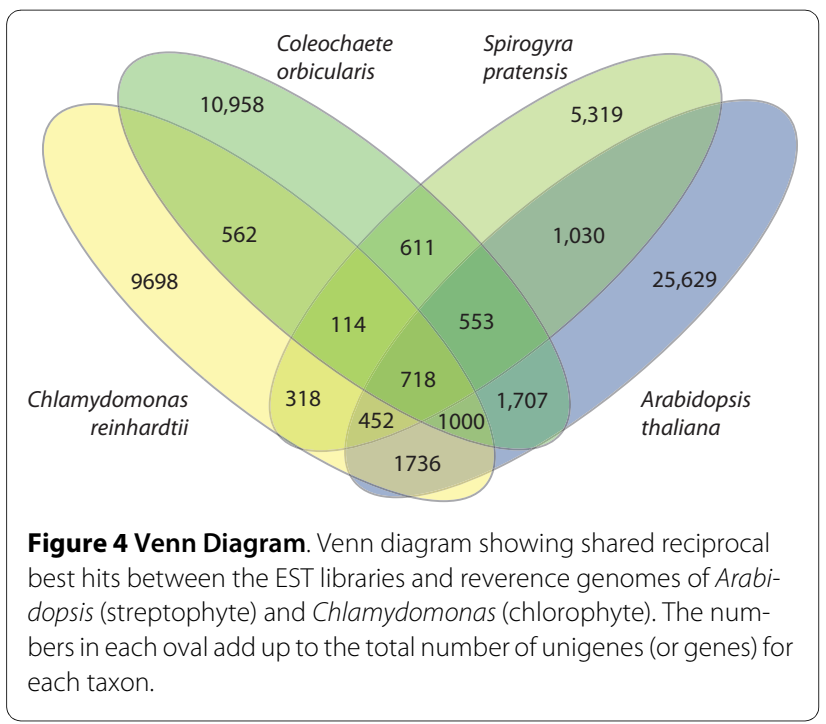

tral moss genome revealed a loss of flagaller associated genes [21] that are found in other green algal and animal genomes[18]. The fact that there are no significant land plant hits suggests that these proteins were lost or underwent substantial sequence divergence in the early land plants. Also interesting is the mere presence of flagellarassociated proteins in Spirogyra, which lacks motile cells entirely. Containing genes for an absent structure further supports the evolutionary placement of this lineage as being derived from flagellate ancestors. An earlier hypothesis had the Zygnematales grouped with other non-flagellate organisms in a separate phylum defined by lack of motility [22], which our data clearly reject.

The overwhelming similarity to land plants also is revealed in a separate analysis in the identification of orthologous genes between our two green algae, Chlamydomonas and Arabidopsis, as shown in the Venn diagram (Fig.4). The charophytes share two to three times as many orthologs with Arabidopsis than they do with Chlamydomonas. Both the BLAST analysis and the ortholog identification reinforce the overall similarity between these lineages of green algae and land plants. Of course, a robust phylogeny of any given gene is needed before firm conclusions can be made regarding its gain or loss along the plant lineage. The point here is that even a simple BLAST analysis or ortholog identification can reveal key genes to target for follow-up study.

There are a large number of putative novel genes in both EST libraries (20\% and 28\% for Spirogyra pratensis and Coleochaete orbicularis, respectively). The term 'novel' is being used here to refer to genes with no obvious similarity to a model-organism sequence: i.e. new to scientists, not necessarily to the charophytes. We are classifying the unigenes as novel if they satisfy two criteria: they contain a protein-coding region predicted by ESTScan [19] and no BLAST hits returned against the NCBI non-redundant protein database. The closest fully sequenced genomes to the charophytes at the time of this publication are several embryophyte genomes, including Physcomitrella patens and Arabidopsis thaliana (which have at least 450 million years divergence with the charophytes) and Chlamydomonas or Ostreococcus in the chlorophyte lineage (which have 700-1,000 million years divergence) [2]. Because of the relative distance between charophytes and their nearest sequenced organisms, it is not surprising that they would contain genes with no obvious similarity to the current published genome-scale sequences. There are two alternative explanations for the enlarged amount of "ESTs without BLAST hits". First, the short sequences may represent non-conserved portions of genes, and, second, independent 454 sequence stretches may correspond to the same novel gene, but they do not overlap due to their limited sequence length. This last caveat would apply across the entire set of clus- 
Table 3: BLAST results for plant genes. Genes hypothesized to be important in the colonization and adaptation of land plants (Graham et al. 2000).

\begin{tabular}{|c|c|c|c|c|c|c|c|c|c|c|}
\hline & \multirow[t]{2}{*}{ Gene name } & \multirow[t]{2}{*}{ Tair Num. } & \multicolumn{2}{|c|}{ P.patens } & \multicolumn{2}{|c|}{ C. reinhartii } & \multicolumn{2}{|c|}{ C. orbicularis } & \multicolumn{2}{|c|}{ S. pratensis } \\
\hline & & & Hits & e-value & Hits & e-value & Hits & e-value & Hits & e-value \\
\hline Cellulosic cell wall & RSW1 & AT4G32410 & 19 & 0.0 & - & - & 2 & e-130 & 5 & 0.0 \\
\hline $\begin{array}{l}\text { Cytokinetic } \\
\text { phragmoplast }\end{array}$ & GEM1/MOR1 & AT2G35630 & 2 & 0.0 & 1 & 0.0 & 2 & e-51 & 1 & $2 e-70$ \\
\hline Plasmodesmata & CRT1 & AT1G56340 & 6 & $e-62$ & 1 & e-125 & 5 & e-141 & 3 & $3 e-53$ \\
\hline $\begin{array}{l}\text { asymmetric cell } \\
\text { division }\end{array}$ & WUSCHEL & AT1G20700 & 2 & e-32 & - & - & 2 & e-33 & 0 & \\
\hline $\begin{array}{l}\text { asymmetric cell } \\
\text { division }\end{array}$ & GNOM & AT1G13980 & 7 & 0.0 & 3 & e-108 & 1 & $7 e-49$ & 0 & \\
\hline Specialized cells & Alpha Expansions & AT1G12560 & 39 & $e-70$ & - & - & 0 & & 12 & $3 e-53$ \\
\hline Specialized cells & Beta Expansoins & AT1G65680 & 4 & $7 e-44$ & - & - & 0 & & 2 & $1 e-24$ \\
\hline $\begin{array}{l}\text { Multicellular } \\
\text { sporophyte body }\end{array}$ & MERISTEM LAYER 1 & AT4G21750 & - & - & - & - & 1 & e-106 & 1 & $6 e-53$ \\
\hline $\begin{array}{l}\text { Ploidy level } \\
\text { influence in tissue } \\
\text { differentiation }\end{array}$ & MADS domain & AT1G22130 & 10 & e-22 & - & - & 0 & & 0 & \\
\hline
\end{tabular}

CDSs of noted genes were BLASTed against protein databases of two reference genomes, Physcomitrella patens and Chlamydomonas reinhartii, and our two charophytes, Coleochaete orbicularis and Spirogyra pratensis. Only BLASTx hits below e-20 are reported.

tered set of ESTs for both species. Aside from these concerns, most of these novel sequences do appear to be unique, at least within the available data. A pair-wise BLAST between the $S$. pratensis and C. orbicularis sets of novel unigenes only revealed a small amount of overlap: out of 2,661 S. pratensis novel genes, only 24 had a hit in C. orbicularis. In addition, the reciprocal BLAST revealed only 27 hits in the reverse direction. None were reciprocal best hits.

The Gene Ontology analysis presents a summary of transcript diversity relative to the annotated gene diversity in Arabidopsis. Where there are significant differences in the proportion of genes in a category, the determination cannot be made whether there were fewer genes expressed or that there are actually fewer genes encoded in the genome--and the same goes for the relative over-expression. The observation that both charophyte green algal ESTs were so proportionally similar in their gene expression categories suggests that we are not missing any major class of gene expression and that we have captured a fairly wide range of transcript diversity representing the source genome's diversity. Spirogyra and Coleochaete are separated in evolutionary time by 500 700 million years and they do not share a similar gross morphology. But the fact that they are so alike in transcript proportion, as compared to a flowering plant, is interesting in terms of their evolutionary placement in the streptophyte lineage. The green algae showed signifi- cant difference with respect to Arabidopsis in most GO categories, but only a few were markedly divergent.

The under representation of charophyte genes in some of the Biological Process categories (Figure 5a) is not surprising. Although biological complexity is not easily defined, it seems clear that embryophytes have a larger number of cell types and are adapted to a wider range of environmental conditions than are charophyte algae, and consequently would be expected to have a higher proportion of genes expressed in categories involving organismal complexity. Both charophytes were significantly underrepresented in their proportion of cellular metabolic process transcripts compared to Arabidopsis. According to the Gene Ontology Consortium's (GOC) description of this category --the chemical reactions and pathways by which individual cells form chemical substances [23]--it stands to reason that the diversity and abundance of transcripts in this class would be higher in an organism with more diverse cell types, such as the flowering plant taxon, Arabidopsis. The same interpretation goes for other underrepresented categories including cell communication, response to endogenous stimulus and regulation of biological quality. The latter category had the most extreme underrepresentation of the Biological Processes categories and consequently may be one of the more interesting categories in the study of the origin of land plants. Biological quality is defined by GOC as "a measurable attribute of an organism or part of an organ- 


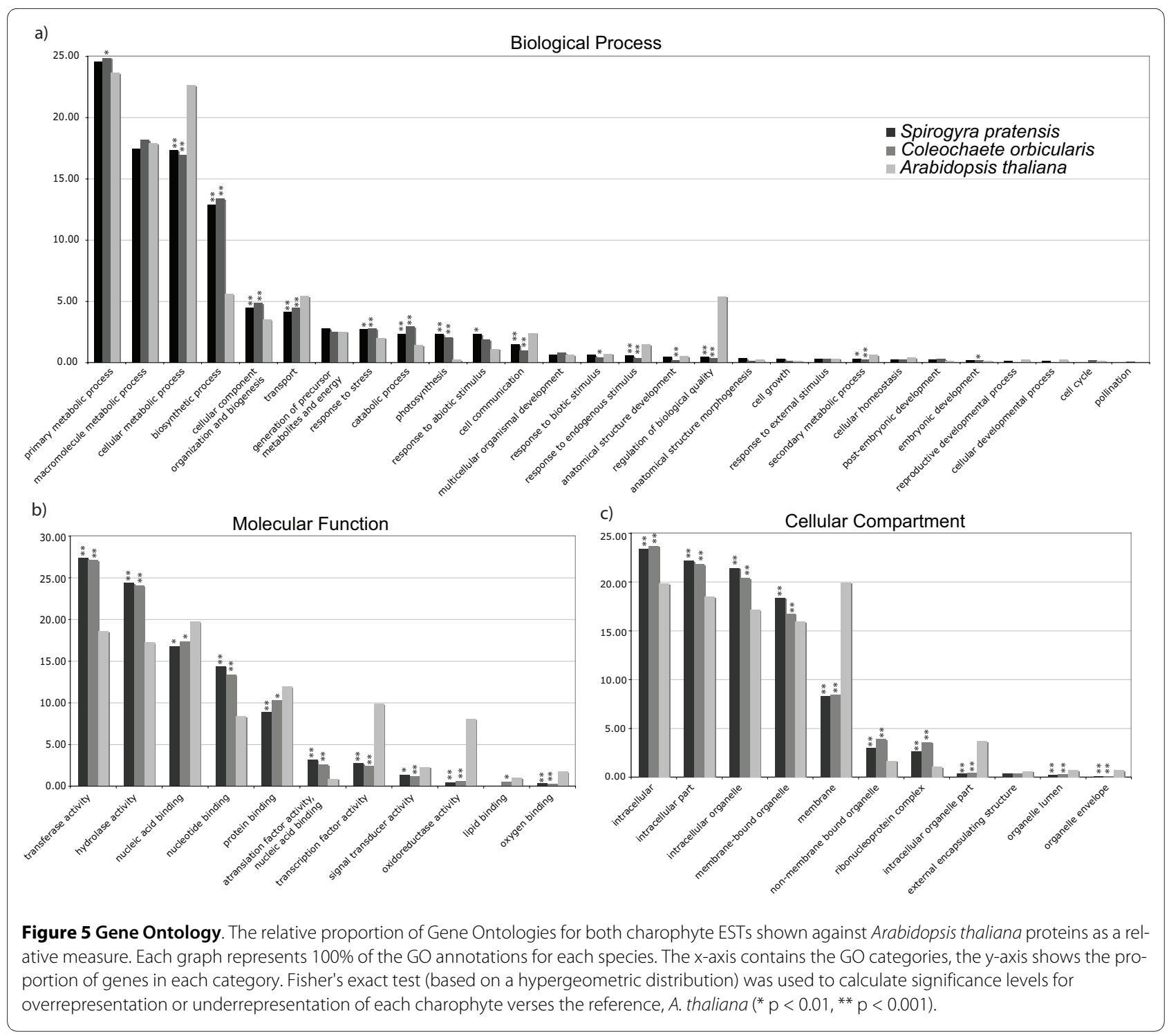

ism, such as size, mass, shape, color, etc." The charophyte transcripts annotated to this category were similar to genes involved in cell shape, such as DIMINUTO [24], and various expansins [25]. It is logical to expect that the proportion of genes associated with cell quality would increase with organism complexity and our results are consistent with this prediction.

The explanation becomes less clear in cases where there is overrepresentation of charophyte genes in a given Biological Process category relative to Arabidopsis. Because we are comparing proportions of genes present and not the actual number found, it is possible that there might be a greater number of genes in Arabidopsis compared to one of the charophytes. But the proportion relative to the other categories is lower in our analysis. That said, one of the more extreme overrepresentations (over twice the proportion) of charophyte genes occurs in the biosynthetic process category, which is defined by "the chemical reactions and pathways resulting in the formation of substances; typically the energy-requiring part of metabolism in which simpler substances are transformed into more complex ones" [23]. Examples of charophyte genes that are annotated in this category are ribosomal proteins, EF-1-alpha-related GTP-binding proteins, replication proteins, translation initiation factors and ATP binding proteins. These are all core functioning genes and therefore are not expected to diversify with increasing complexity. It is likely the case that the charophyte transcriptomes contain a larger proportion of core functioning genes when compared to Arabidopsis, simply because they are lacking diversity in the other classes. Another way to look at it is that Coleochaete and Spirogyra cells are analogous to plant parenchyma and are enriched for the kinds of metabolic processes that occur in parenchyma, and not due to any trend towards increasing diversity of genes in charophyte biosynthetic pro- 
cesses. This probably also holds true in the photosynthesis category, where both charophytes show a significant overrepresentation of genes relative to Arabidopsis. An alternative hypothesis would be that, lacking structural complexity, charophytes have evolved a broader range of biochemical responses to the environment. As with most EST studies, we cannot rule out the possibility that the apparent over-representation might be a sampling artifact due to non-normalized cDNA or incomplete coverage.

Ontology by Cellular Compartment (Figure 5c) reveals one extreme difference in the membrane category whereby the charophytes are extremely underrepresented. It follows that parenchymatous tissue (i.e. the plant tissue of Arabidopsis) would involve an increased level of cell-to-cell communication requiring many more membrane proteins than would a filamentous organism, like Spirogyra, or a two-dimensional disk-shaped form, like Coleochaete.

Ultimately, we are interested in specific genes that allowed a lineage of charophytes to colonize land so successfully and give rise to land plants. Because the experimental approach was a survey, and because the work reported here constitutes just a part of the overall effort to gather transcriptome data for all of the charophyte orders, we are highlighting genes of interest to follow up for further study. It is encouraging that most of the genes identified by Graham et al. [20] as likely to have been important in the colonization of the land have solid hits and perhaps even true orthologs in both Coleochaete orbicularis and Spirogyra pratensis (Table 3). A couple of the more interesting genes associated with asymmetric cell division (WUSCHEL and GNOM) have hits in Coleochaete, but not Spirogyra. This is consistent with the morphology of the organisms; Spirogyra, an unbranched, filamentous alga does not have asymmetric cell division, but Coleochaete definitely does. No significant BLAST hits of the Graham et al. genes were found in the ESTs of the earliest diverging streptophyte, Mesostigma virde. Another interesting finding is the presence of ethylene biosynthesis and signalling pathway genes (Table 4), long thought to be unique to land plants. This finding suggests that ethylene might be utilized in the charophytes. Wang et al. [26] showed that Chara spp. (also a charophyte) showed ethylene binding activity similar to that of land plants, so it is not too surprising that these genes should be present in other charophyte lineages. However, to the best of our knowledge, evidence of ethylene-signalling genes in charophytes has not been shown before this study.

\section{Conclusions}

We analyzed EST data from two species of charophyte green alga, Spirogyra pratensis and Coleochaete orbicu- laris as a first step toward complete genome analyses. Algal cultures were grown and collected in various life stages as well as at different times during the day. All of these factors were part of an effort to maximize the total number of transcripts available for each pulled EST library. We combined both Sanger and 454 sequencing technologies to obtain as much sequence coverage as possible. Both of the resulting EST libraries gave a nice diversity of transcripts (unigenes), with the 454 sequences contributing to most of that transcript diversity. This is especially pronounced when looking at the sequencing source of the singletons: Sanger reads only contributed a small fraction of the total singletons in both EST libraries (Table 2), and the 454 data alone covered most of the sequences obtained by Sanger sequencing. Although there have been just a few 454 transcriptome sequencing efforts to date [27-31], our results suggest that for studies of this type, 454 sequencing alone could largely replace traditional Sanger Sequencing. In summary, the specific genes we mention in this paper (Tables 3 and 4), along with the Gene Ontology analysis and ortholog identification, reinforce our finding of the overall genomic similarity that these two charophytes have to land plants.

\section{Methods}

\section{Algal sampling and material collection}

The following criteria were used for our taxon sampling: the availability of cultures and ease of cultivation, the presence of a sizable research community, the history of research with this organism, the perceived potential for development of advanced molecular tools, and their key evolutionary positions in two major charophyte orders. Spirogyra pratensis Transeau (UTEX LB 928) (Zygnematales) and Coleochaete orbicularis Pringsh. (UTEX LB 2651) (Coleochaetales) were grown up in Guillard's Woods Hole medium [32] at $18^{\circ} \mathrm{C}$ and a 12:12 LD photoperiod with a photon flux of $180-200 \mu \mathrm{mol} \mathrm{s}^{-1} \mathrm{~m}^{-2}$. Cultures were harvested during log phase growth in a variety of conditions to maximize the diversity of transcripts: at intervals at $7 \mathrm{am}, 12 \mathrm{pm}, 4 \mathrm{pm}$ and $9 \mathrm{pm}$; after sitting in a dark enclosure for 24 hours; and after being exposed to $20 \mathrm{~min}$ of $-20^{\circ} \mathrm{C}$. Spirogyra pratensis filaments were removed from the medium using a sterile glass hook, wicked to remove excess moisture, dropped in liquid nitrogen and stored at $-80^{\circ} \mathrm{C}$ until RNA extraction. Coleochaete orbicularis cultures were scraped from the culturing flask, pelleted at 4000rpm, dropped in liquid nitrogen and stored at $-80^{\circ} \mathrm{C}$ until RNA extraction.

\section{RNA isolation}

Frozen tissue was ground at cryogenic temperatures using a SPEX 6770 Freezer/Mill (SPEX Certi Prep, Metuchen, NJ). The frozen ground tissue was then added 
Table 4: BLAST results for ethylene pathway genes. Genes involved in ethylene biosynthesis and the ethylene response pathway.

\begin{tabular}{|c|c|c|c|c|c|c|}
\hline \multirow[t]{2}{*}{ Gene function } & \multirow[t]{2}{*}{ Gene name } & \multirow[t]{2}{*}{ Tair Num. } & \multicolumn{2}{|c|}{ C. orbicularis } & \multicolumn{2}{|c|}{ S. pratensis } \\
\hline & & & BLAST hit & e-value & BLAST hit & e-value \\
\hline Ethylene biosynthesis & ACO1 & AT2G19590.1 & 1 & $4 e-30$ & 0 & \\
\hline Ethylene biosynthesis & ACS5 & AT1G62960.1 & 1 & $2 e-33$ & 1 & $3 e-32$ \\
\hline Ethylene binding & ETR1 & AT1G66340.1 & 0 & & 5 & $e-101-2 e-23$ \\
\hline Ethylene binding & ERS1 & AT2G40940.1 & 0 & & 3 & $2 e-61-6 e-41$ \\
\hline Ethylene binding & ETR2 & AT3G23150.1 & 0 & & 2 & $2 e-36-8 e-29$ \\
\hline Ethylene binding & EIN4 & AT3G04580.1 & 0 & & 5 & $8 e-51-3 e-21$ \\
\hline Ethylene pathway & CTR1 & AT5G03730.2 & 5 & $2 e-73-1 e-43$ & 5 & $9 e-80-1 e-42$ \\
\hline Ethylene pathway & EIN2 & AT5G03280.1 & 1 & $1 e-18^{*}$ & 0 & \\
\hline Transcription factor & EIN3 & АT3G20770.1 & 1 & $2 \mathrm{e}-73$ & 1 & e-128 \\
\hline Transcription factor & ERF1 & AT5G47880.1 & 2 & $0.0-4 e-43$ & 1 & e-147 \\
\hline Ethylene pathway & EBF1 & AT2G25490.1 & 1 & $9 e-73$ & 1 & $3 e-94$ \\
\hline Ethylene pathway & RTE1 & AT2G26070.1 & 0 & & 0 & \\
\hline
\end{tabular}

* indicates the ethylene binding domain (EBD) is present in the BLAST hit.

to $2 \times$ RNA extraction buffer as described in La Claire and Herrin [33], in which case a modified version of this protocol for RNA isolation was followed. After each isolation, the nucleic acid concentration and OD ratios (260/ 280 and 260/230) were quantified with a NanoDrop (Thermo Scientific NanoDropTM 1000 Spectrophotometer, Wilmington, DE) and the quality of RNA, or the degree of degradation, was determined by running $1 \mu \mathrm{g}$ of total RNA on a $1.2 \%$ agarose MOPS/formaldehyde gel (Applied Biosystems/Ambion, Austin, TX) stained with ethidium bromide, then examining the rRNA banding patterns. High-quality, clean RNA was pooled until $1 \mathrm{mg}$ of total RNA was reached.

\section{cDNA construction}

Total RNA (1 mg) was shipped on dry ice to Agencourt Bioscience Corporation (Beverly, MA) where Poly(A)+RNA from total RNAs was isolated by two rounds of oligo $(\mathrm{dT})$ selection with oligo(dT) coated magnetic particles (Seradyn, Inc.). From the poly(A)+RNA, cDNA libraries were constructed by using an oligo dT primer-adapter containing a Not I site and Moloney Murine Leukemia Virus Reverse Transcriptase (M-MLV $\mathrm{RT}$ ) to prime and synthesize first strand cDNA. After the second strand was synthesized, the double stranded cDNA was size fractionated $(<1.2 \mathrm{~kb})$, cloned directionally into the pExpress 1 vector and grown up in T1 phage resistant E. coli.

\section{DNA sequencing}

DNA sequencing for Spirogyra pratensis and Coleochaete orbicularis included both 5 prime Sanger reads and 454 sequencing technologies: S. pratensis had a targeted 10,000 Sanger read plus a full plate of GS FLX Standard 454 sequences generated; $C$. orbicularis had a targeted 5,000 Sanger reads plus a full plate of GS FLX Titanium 454 sequences generated.

\section{Sanger sequencing}

DNA from the clones was purified using Agencourt's proprietary large-scale automated template purification systems using solid-phase reversible immobilization (SPRI). The purified DNA was then sequenced using ABI dye-terminator chemistry and then run on $\mathrm{ABI} 3730 \times 1$ (Applied Biosystems Inc, Foster City, CA) machines.

\section{4-sequencing}

3-5ug of isolated DNA was nebulized to a mean size range of 3-500 bp, followed by a size selection of fragments $>300$ bp by column exclusion and Ampure ${ }^{\mathrm{Tm}}$ (Agencourt Bioscience, Danvers, MA) isolation. The correct size selection was confirmed on an Agilent DNA 1000 LabChip. Adapters were ligated onto the fragments and selected using library capture beads. The single stranded fragments were isolated with $0.125 \mathrm{~N} \mathrm{NaOH}$, followed by neutralization with acetic acid, and purified. Single stranded library was validated qualitatively by Agilent RNA Pico 6000 LabChip and quantitatively by Invitrogen Ribogreen assay. Standard library dilutions were made according to published protocol. The library was amplified onto DNA capture beads by emulsion PCR (emPCR). DNA capture beads were collected by washes with isopropanol, Roche 454 emPCR collection reagents, and filtered syringes. Sequencing primer was annealed by thermocycler. Collected beads were quantified by count- 
ing on Beckman Multisizer. Beads for each genome were placed on the picotitre plate, sequenced on the Roche 454 GS FLX instrument, and analysed with base-calling software using default parameters.

\section{EST Analysis}

The clustering was preformed in a two-step process. First, 454 library construction adapter sequences were trimmed, then the reads were clustered using MIRA vs $2.9 .43[34,35]$ with $454 \mathrm{EST}$ assembly specific parameters. Second, the raw Sanger reads were combined with the 454 contigs + singleons (along with their respective quality scores) and were cleaned and clustered using the EST2uni pipeline [36]. (See Fig. 1) This pipeline used Phred [37,38], SeqClean [39], Lucy [40], and RepeatMasker [41] to remove low-quality sequence, vector contamination and low complexity regions. It then clustered the clean reads with CAP3 [42] using a 100 bp plus 95 percent identity of overlap. ESTscan [19] predicted the protein-coding regions in the contigs and singletons using the score matrix from Arabidopsis thaliana. At this point, the unigenes (contigs plus singletons) were available in three forms: raw nucleotides, predicted amino acids and nucleotide coding sequences (cds). Annotation of the unigenes was determined in a variety of ways. To screen for contamination, we BLASTed nucleotide unigenes against NCBI's non-redundant protein database (nr) (evalue $<0.0001$ ), producing a lineage assignment for each EST. Taxonomic ID and lineage information for each BLAST hit was retrieved from NCBI Entrez using inhouse perl scripts. Gene annotation was performed by BLASTing the predicted amino acid unigenes against the model plant, A. thaliana (TAIR8) (e-value $<10-6$ ). The top hit was extracted with an in-house perl script and used for the EST unigene annotation.

The putative orthologs between our two charophytes, Spirogyra and Coleochaete, a land plant, Arabidopsis thaliana, and a chlorophyte, Chlamydamonas reinhartii were determined using the reciprocal best hit $(\mathrm{RBH})$ criterion [43]. An all-by-all BLASTp (e-value $\left.<1 \mathrm{e} \times 10^{-6}\right)$ was preformed on the proteins from each of the four taxa. In house perl scripts were used to parse the blast hits, extract the RBH pairs, and expand the pairs to include $\mathrm{RBHs}$ for multiple genomes/transcriptoms. The RBHs between any two, three or four species were interpreted to be orthologs if all pairwise possibilites in the ortholog set were each other's reciprocal best BLAST hit.

The BLAST results against Arabidopsis thaliana were also used for Gene Ontology (GO) annotation by importing the $x \mathrm{xml}$ BLAST results into the program Blast2GO [44]. Gene Ontology assignments were performed as follows: first, associated GO terms were mapped to the top hits of the BLAST search (against $A$. thaliniana) and, by extension, to the EST unigene; select GO annotations were chosen from the mapped group of GO terms; and finally, a combined graph analysis for Biological Processes, Molecular Function and Cellular Compartment resulted in a level three GO distribution. Arabidopsis thaliana GO analysis was included in the bar graph for reference and a Fisher's exact test (based on a hypergeometric distribution) was used to calculate significance levels for overrepresentation or underrepresentation of each charophyte vs. the reference, $A$. thaliana.

Arabidopsis genes of interest in early land plant evolution were obtained from http://www.arabidopsis.org/ then BLASTed against both Coleochaete orbicularis and Spirogyra pratensis (BLASTx, e-value $<1 \mathrm{e}-20$ ). The number of BLASTx hits with e-values below this threshold were considered possible homologs for the plant gene of interest.

The individual reads comprising each EST library were deposited in GenBank. The Sanger reads are located in $\mathrm{dbEST}$ under the following accession numbers: Coleochaete orbicularis (GenBank: GW591203-GW595666), Spirogyra pratensis (GenBank: GW595667-GW602960). The 454 sequences are in the Sequence Read Archive (SRA): C. orbicularis (GenBank: SRX017046.2), S. pratensis (GenBank: SRX017045.2). The clustered ESTs are available for download on the author's webpage [45].

\section{Additional material}

Additional file $1 \mathrm{RBH}$ ortholog sets for charophytes + Chlamydomo-

nas. Reciprocal Best Hit sets of unigenes that are shared between the

Charophytes and Chlamydomonas, but which are absent in Arabidopsis. chlre: Chlamydomonas reinhartii; corb: Coleochaete orbicularis; spra: Spirogyra pratensis.

\section{Authors' contributions}

RET cultured the algae, carried out the wet bench work, built the EST analysis pipeline and drafted the manuscript. CFD participated in the design and coordination of the study and helped to draft and edit the manuscript. Both authors read and approved the final manuscript.

\section{Acknowledgements}

The study was supported by NSF (Division of Molecular and Cell Biosciences, Microbial genome Sequencing Program), MCB-0523719. Special thanks go to Cristian Castillo-Davis for his advice on the GO statistics, Jennifer Bendery for her careful editing and to the reviewers whose comments greatly improved the final manuscript.

\section{Author Details}

${ }^{1}$ Cell Biology and Molecular Genetics, University of Maryland, College Park, MD, 20742, USA and ${ }^{2}$ Cell Biology and Molecular Genetics, Maryland Agricultural Experiment Station, University of Maryland, College Park, College Park, MD 20742, USA

Received: 20 November 2009 Accepted: 25 May 2010

Published: 25 May 2010

\section{References}

1. Kenrick P, Crane PR: The origin and early evolution of plants on land. Nature 1997, 389:33-39.

2. Zimmer A, Lang D, Richardt S, Frank W, Reski R, Rensing SA: Dating the early evolution of plants: detection and molecular clock analyses of orthologs. Mol Genet Genomics 2007, 278(4):393-402.

3. Karol KG, McCourt RM, Cimino MT, Delwiche CF: The closest living relatives of land plants. Science 2001, 294(5550):2351-2353. 
4. Lewis $L A, M c C o u r t ~ R M:$ Green algae and the origin of land plants. Am J Bot 2004, 91:1535-1556.

5. Lemieux C, Otis C, Turmel M: A clade uniting the green algae Mesostigma viride and Chlorokybus atmophyticus represents the deepest branch of the Streptophyta in chloroplast genome-based phylogenies. BmC Biol 2007, 5:

6. Rodriguez-Ezpeleta N, Philippe H, Brinkmann H, Becker B, Melkonian M: Phylogenetic analyses of nuclear, mitochondrial, and plastid multigene data sets support the placement of Mesostigma in the Streptophyta. Mol Biol Evol 2007, 24:723-731.

7. Turmel M, Ehara M, Otis C, Lemieux C: Phylogenetic relationships among streptophytes as inferred from chloroplast small and large subunit rRNA gene sequences. J Phycol 2002, 38(2):364-375.

8. Turmel M, Pombert JF, Charlebois P, Otis C, Lemieux C: The green algal ancestry of land plants as revealed by the chloroplast genome. Int $\mathrm{J}$ Plant Sci 2007, 168(5):679-689.

9. Mattox KR, Stewart KD: A classification of the green algae: a concept based on comparative cytology. In Systematics of the Green Algae Edited by: Irvine DEG, John DM. London UK: Academic Press; 1984:29-72.

10. Simon A, Gloeckner G, Felder M, Melkonian M, Becker B: EST analysis of the scaly green flagellate Mesostigma viride (Streptophyta): Implications for the evolution of green plants (Viridiplantae). BMC Plant Biol 2006, 6:

11. Wodniok S, Simon A, Gloeckner G, Becker B: Gain and loss of polyadenylation signals during evolution of green algae. BMCEvol Biol 2007, 7:

12. Turmel M, Otis C, Lemieux C: An unexpectedly large and loosely packed mitochondrial genome in the charophycean green alga Chlorokybus atmophyticus. Bmc Genomics 2007, 8:137.

13. Noble GP, Rogers MB, Keeling PJ: Complex distribution of EFL and EF-1 alpha proteins in the green algal lineage. Bmc Evo Biol 2007, 7:82.

14. Grauvogel C, Petersen J: Lsoprenoid biosynthesis authenticates the classification of the green alga Mesostigma viride as an ancient streptophyte. Gene 2007, 396:125-133.

15. Turmel M, Otis C, Lemieux C: The chloroplast and mitochondrial genome sequences of the charophyte Chaetosphaeridium globosum: Insights into the timing of the events that restructured organelle DNAs within the green algal lineage that led to land plants. Proc Natl Acad Sci USA 2002, 99(17):11275-11280

16. Nakayama T, Marin B, Kranz H, Surek B, Huss V, Inouye I, Melkonian M: The basal position of scaly green flagellates among the green algae (Chlorophyta) is revealed by analyses of nuclear-encoded SSU rRNA sequences. Protist 1998, 149(4):367-380.

17. Derelle E, Ferraz C, Rombauts S, Rouze P, Worden AZ, Robbens S, Partensky F, Degroeve S, Echeynie S, Cooke R, et al.: Genome analysis of the smallest free-living eukaryote Ostreococcus tauri unveils many unique features. Proc Natl Acad Sci USA 2006, 103(31):11647-11652.

18. Merchant SS, Prochnik SE, Vallon O, Harris EH, Karpowicz SJ, Witman GB, Terry A, Salamov A, Fritz-Laylin LK, Marechal-Drouard L, et al: The Chlamydomonas genome reveals the evolution of key animal and plant functions. Science 2007, 318(5848):245-251.

19. Iseli C, Jongeneel CV, Bucher P: ESTScan: a program for detecting, evaluating, and reconstructing potential coding regions in EST sequences. Proceedings: International Conference on Intelligent Systems for Molecular Biology 1999:138-148.

20. Graham LE, Cook ME, Busse JS: The origin of plants: Body plan changes contributing to a major evolutionary radiation. Proc Natl Acad Sci USA 2000, 97:4535-4540.

21. Rensing SA, Lang D, Zimmer AD, Terry A, Salamov A, Shapiro H, Nishiyama $T$, Perroud $P$, Lindquist EA, Kamisugi Y, et al: The Physcomitrella genome reveals evolutionary insights into the conquest of land by plants. Science 2008, 319(5859):64-69.

22. Margulis L, Schwartz KV: Five Kingdoms: An illustrated guide to the phyla of life on earth. New York: W. H. Freeman and Company; 1988

23. Ashburner M, Ball CA, Blake JA, Botstein D, Butler H, Cherry JM, Davis AP, Dolinski K, Dwight SS, Eppig JT, et al:: Gene Ontology: tool for the unification of biology. Nat Genet 2000, 25:25-29.

24. Takahashi T, Gasch A, Nishizawa N, Chua NH: The DIMINUTO gene of Arabidopsis is involved in regulating cell elongation. Genes Dev 1995, 9(1):97-107

25. Cosgrove DJ: Loosening of plant cell walls by expansins. Nature 2000 407(6802):321-326
26. Wang W, Esch JJ, Shiu S, Agula H, Binder BM, Chang C, Patterson SE, Bleecker AB: Identification of important regions for ethylene binding and signaling in the transmembrane domain of the ETR1 ethylene receptor of Arabidopsis. Plant Cell 2006, 18(12):3429-3442.

27. Cheung F, Haas BJ, Goldberg SMD, May GD, Xiao Y, Town CD: Sequencing Medicago truncatula expressed sequenced tags using 454 Life Sciences technology. Bmc Genomics 2006, 7:272.

28. Zou Z, Najar F, Wang Y, Roe B, Jiang H: Pyrosequence analysis of expressed sequence tags for Manduca sexta hemolymph proteins involved in immune responses. Insect Biochem Mol Biol 2008 38(6):677-682

29. Rothberg JM, Leamon JH: The development and impact of 454 sequencing. Nat Biotechnol 2008, 26(10):1117-1124.

30. Vera JC, Wheat CW, Fescemyer HW, Frilander MJ, Crawford DL, Hanski I Marden JH: Rapid transcriptome characterization for a nonmodel organism using 454 pyrosequencing. Mol Ecol Notes 2008, 17(7):1636-1647.

31. Cheung F, Win J, Lang JM, Hamilton J, Vuong H, Leach JE, Kamoun S, Levesque CA, Tisserat N, Buell CR: Analysis of the Pythium ultimum transcriptome using Sanger and Pyrosequencing approaches. Bmc Genomics 2008, 9:542.

32. Guillard RL, Keller MD: Culturing dinoflagellates. In Dinoflagellates Edited by: Spector DL. San Diego, CA: Academic Press; 1984:391-442.

33. LaClaire JW, Herrin DL: Co-isolation of high-quality DNA and RNA from coenocytic green algae. Plant Molecular Biology Reporter 1997, 15:263-272.

34. Chevreux B, Pfisterer T, Drescher B, Driesel AJ, Muller WEG, Wetter T, Suhai $\mathrm{S}$ : Using the miraEST assembler for reliable and automated mRNA transcript assembly and SNP detection in sequenced ESTs. Genome Res 2004, 14(6):1147-1159.

35. Chevreux B, Wetter T, Suhai S: Genome Sequence Assembly Using Trace Signals and Additional Sequence Information. Computer Science and Biology: Proceedings of the German Conference on Bioinformatics (GCB) 1999, 99:45-56

36. Forment J, Gilabert F, Robles A, Conejero V, Nuez F, Blanca JM: EST2uni: an open, parallel tool for automated EST analysis and database creation, with a data mining web interface and microarray expression data integration. BMC Bioinformatics 2008, 9:5.

37. Ewing B, Green P: Base-calling of automated sequencer traces using phred. II. Error probabilities. Genome Res 1998, 8(3):186-194.

38. Ewing B, Hillier L, Wendl MC, Green P: Base-calling of automated sequencer traces using phred. I. Accuracy assessment. Genome Res 1998, 8(3):175-185.

39. TIGR Seqclean Tool [http://compbio.dfci.harvard.edu/tgi/software/]

40. Chou HH, Holmes MH: DNA sequence quality trimming and vector removal. Bioinformatics 2001, 17(12):1093-1104.

41. Smit A, Hubley R, Green P: RepeatMasker Open-3.0. 1996 [http:// www.repeatmasker.org].

42. Huang X, Madan A: CAP3: A DNA Sequence Assembly Program. Genome Res 1999, 9:868-877.

43. Tatusov RL, Koonin EV, Lipman DJ: A genomic perspective on protein families. Science 1997, 278(5338):631-637.

44. Conesa A, Gotz S, Garcia-Gomez JM, Terol J, Talon M, Robles M: Blast2GO: a universal tool for annotation, visualization and analysis in functional genomics research. Bioinformatics 2005, 21:3674-3676.

45. [http://www.ruthtimme.com]

doi: 10.1186/1471-2229-10-96

Cite this article as: Timme and Delwiche, Uncovering the evolutionary origin of plant molecular processes: comparison of Coleochaete (Coleochaetales) and Spirogyra (Zygnematales) transcriptomes BMC Plant Biology 2010, 10:96 